\title{
Fabrication and Evaluation of Rotary Separator of Sesame Oil
}

\author{
Abbas Akbarnia*, Mahdi Rashvand \\ Machine Design and Mechatronics Department, Institute of Mechanics, Iranian Research Organization for Science and Technology, Tehran, \\ Iran
}

Email address:

abbasakbarnia@irost.it (A. Akbarnia)

${ }^{*}$ Corresponding author

To cite this article:

Abbas akbarnia, Mahdi Rashvand. Fabrication and Evaluation of Rotary Separator of Sesame Oil. International Journal of Food Science and Biotechnology. Vol. 3, No. 3, 2018, pp. 77-82. doi: 10.11648/j.ijfsb.20180303.11

Received: July9, 2018; Accepted: July 20, 2018; Published: August 16, 2018

\begin{abstract}
Sesame seeds is one of the most important and oldest oily seeds used today in various industries. Sesame oil is also rich in protein substances, which is why it is considered as a quality and valuable oil. In this research, the effect of two angular velocity factors on three levels of 3500, 4500 and $5500 \mathrm{rpm}$ and two levels of the radius, 175 and $125 \mathrm{~mm}$ was investigated in order to investigate on the quality of extracted sesame oil. In this experiment, the cylinder's time was selected for a constant test and equal to 5 minutes. The quality of the oil output from the device was evaluated by a pectometer method and the results were analyzed. The lowest and highest amount of suspended solids was obtained at a speed of $5500 \mathrm{rpm}$ with a cylindrical radius of $175 \mathrm{~mm}$ and a velocity of $3500 \mathrm{rpm}$ with a cylinder with a radius of $125 \mathrm{~mm}$, respectively. The radius of the rotation and cylinder's angular momentum on the gross particles in the refined oil was significant at $1 \%$ level, but the effect of repetition on them was not affected. Also, the interaction between the angular velocity of the cylinder and the radius of cylinder are non-significant.
\end{abstract}

Keywords: Sesame Oil, Decanter, Quality, Velocity, Radius

\section{Introduction}

Sesame is one of the most important oily seeds in some countries. Sesame is a nutrient that is widely used in cereals, especially confectionery products [1] Sesame is free from undesirable pigments, undesirable compounds and antitrypsin. Sesame protein contains globulin $(67.3 \%)$, glutylene (7\%) and prolamin (1.4\%) [2]. According to FAO, in 2014, sesame seeds production was 6235,530 tonnes, of which $59.8 \%$ were produced in Africa and $36.6 \%$ in Asia. Most of the sesame producers in the world are Tanzania with 1138,930 tons, India with 811,000 tons, and Sudan with 721,000 tons.

Sesame oil is known as a price and high quality oil. This oil has a long and lasting resistance to high-fat unsaturated edible oils [3]. The natural antioxidants in this oil have a high resistance to oxidation and valuable physiological properties of sesame oil [4]. Sesame seeds contain $30-50 \%$ oil and 20 $30 \%$ protein [5]. Sesame oil is one of the unsaturated and useful oils for humans, and it has been proven that this oil can lower human cholesterol levels [6].

In the technology of edible oils, extraction of oils and fats from plant and animal resources is a specialized branch. Diversification in oil extraction technology is often due to the diversification of its supply sources, whether plant or animal [7]. In all processes of edible oil extraction, the ultimate goal is to produce healthy, pure and high-yielding oils in a way that the residual oil is a low-fat and valuable product [8]. Different mechanical and chemical methods for extracting oil from these seeds but the most appropriate method is to minimize damage to the oil composition of the seed. In this regard, the use of a mechanical method (press) with the pressure on the texture of these seeds, which leads to the removal of oil in the tissue of these seeds, is particularly noteworthy.

Nowadays, new methods for oil extraction are used to increase the process efficiency in addition to reducing production costs. These methods include mechanical extraction, solvent doping [9], roughingery by mechanical 
method, and Solvent Extraction by Soxhlet [10], Extraction by Superconducting [11], Microwave Extraction [12], extraction by supercritical fluid [13].

In 2011, Pardlahan et al investigated a conical sesame continuous oiling machine. They reported that as much as the exit area of the meal was tighter, more oil was extracted. In other words, the percentage of oil in the meal decreases, but the efficiency of the machine decreases [14]. Also, Beerens (2007) in a study on two continuous press machines found that increasing the spiral rotation speed reduces the oil yield, while the slope of the feed rate increases more rapidly. increasing the spiral speed reduces the pressure and increases the relative temperature of the cylinder [15].

Also, in recent years, using some solvents was also used to extract sesame oil. This method is performed by an extruder and using chemical solvents such as hexane, heptane, diatylate, acetone, trichlorethylene, tetrachlorethylene, ethanol (mostly hexane). The solids are eventually treated with a new solvent and then rinsed. after passing through several stages of oil extraction in the machine [16].

Sarkis et al (2015) performed a research about Application of pulsed electric fields and high voltage electrical discharges for oil extraction from sesame seeds. The objective of this work was to evaluate two different electrical technologies: pulsed electric fields (PEF) and high voltage electrical discharges (HVED), as pretreatments to oil extraction. PEF treatment increased the oil yield by $4.9 \%$ and HVED, if accounted oil losses in water, resulted in the extraction of $22.4 \%$ more oil when samples are compared to control [17].

According to research on sesame oiling by sesame oil extraction machines, it can be claimed that vertical separators are widely used in this industry. Vertical machine operator, due to its structure, is capable of separating mixtures that have a constituent material close to each other. The purpose of this study was to develop sesame oil extraction machine and evaluate the effect of angular velocity and cylinder radius on the quality of refined sesame oil.

\section{Material and Method}

The basis for the separation of materials from centrifugal machines is based on rotational velocity and centrifugal force. Due to the rotation of the centrifugal cylinder, the injected material is also accelerated and separated by centrifugal force from the center of the heavier material (outside the center) and based on the specific gravity of each other. They get This property can be used to separate and treat various mixtures such as oil and dust.

\subsection{Device Layout}

According to the comparison of centrifugal machines with each other, the design of a vertical centrifugal machine (Decanter) was carried out in order to isolate and treat oil from dust Sesame seed presses. In the schematic, the mixture of oil and dust from the central part of the top plate is inserted into the cylinder chamber and accelerated by the impact of the centrifugal force and separated into two solid and liquid phases. The separated oil is transferred to the center of the conductor and transported through the duct in the central shaft (bottom and center). The solids stick to the cylinder wall and drains out of the cylinder after stopping the device (Figure 1).

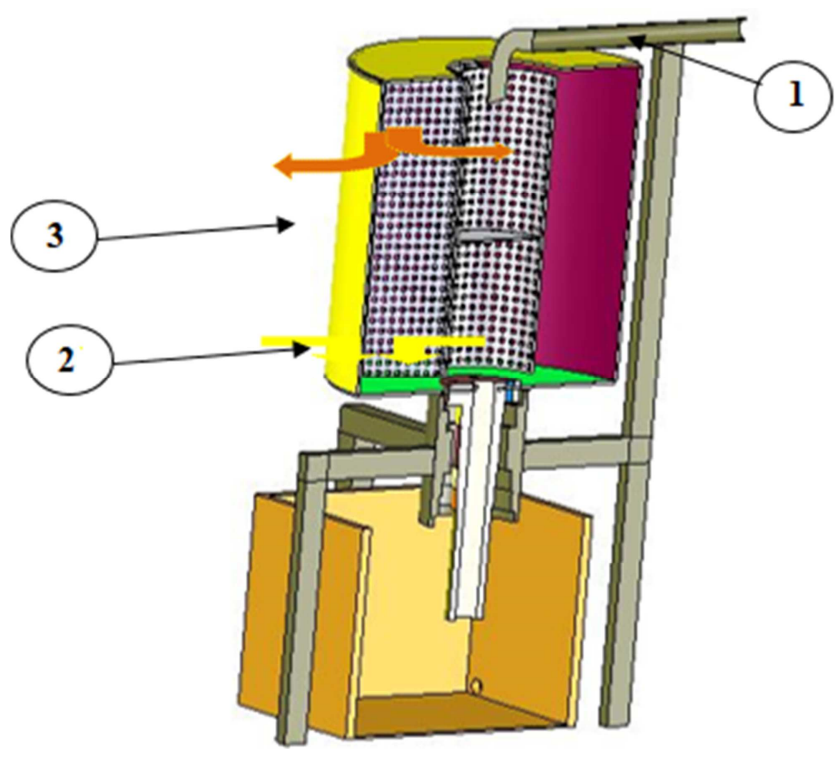

1-Entry of mixed ingredients, 2- Exit flattened oil, 3- Grid net

Figure 1. Separator side section.

Centrifugal force exerted a direct relation with the centrifugal units with their rotation velocity squares, their radius, and their molecular mass. In terms of finding the amount and determining the required rotational speed for the cylinder of the separator, several laboratory tests were carried out using laboratory centrifugal machines. In this experiment, using a J2-21 Centrifuge machine, the Beckman model (Figure 2) and pouring the specified amount of combination of pomace and sesame oil into its rotor tubes relative to the cylinder's cycle, at several times at 2000-5000 rpm In a minute, 500 rounds were played each time for 5 minutes.

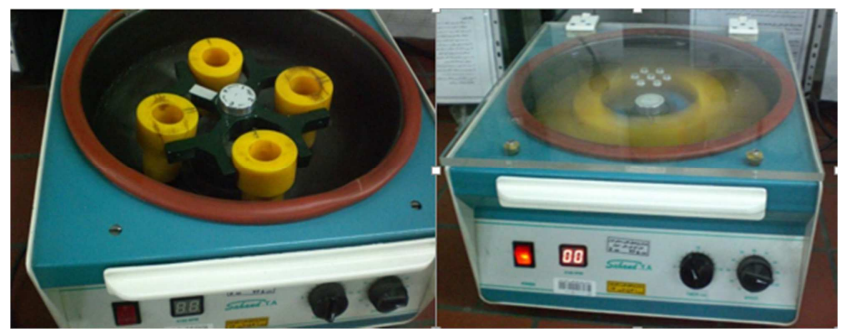

Figure 2. Laboratory centrifuge machine.

Based on the results of the experiments, it was concluded that during separation of 2000 to 3000 rotors the separation operation was not well achieved. Therefore, with an increase of 3,500 rpm for the rotor, it was observed that the separation of the oil was acceptable and when more rotor speed was added, the better the separation and the transparency of the oil were achieved. 


\subsection{Calculate the Power of the Rotary Pump}

The weight of the various components of the decanter includes the cylinder, the gird net, the upper and lower axis were about 18 kilograms.

$$
\mathrm{F}=\mathrm{mg}=18 \times 9.81=176.58 \mathrm{~N}
$$

$\mathrm{F}=$ Decanter displacement weight $(\mathrm{N})$

$\mathrm{m}=$ Decanter weight $(\mathrm{Kg})$

$\mathrm{g}=$ Gravity

The decanter has rotational speed of about $5000 \mathrm{rpm}$, so:

$$
\mathrm{T}=\mathrm{F} \times \mathrm{r}=176.58 \times 0.06=10.6 \mathrm{~N} . \mathrm{m}
$$

$\mathrm{T}=$ the torque from rotation of decanter (N.m)

$\mathrm{r}=$ Radius of rotation $(\mathrm{m})$

Also;

$$
\mathrm{P}=\mathrm{T} . \omega=10.6 \times(2 \times 3.14 \times 5000 / 60)=5544.6 \mathrm{~W}
$$

$\mathrm{P}=$ Rotation power $(\mathrm{W})$

$\omega=$ Radius velocity $(\mathrm{Rad} / \mathrm{s})$

\subsection{Calculate the Diameter of the Axis of the Decanter}

The weight of the decanter and the injected mixture is about $23.9 \mathrm{Kg}$ which can be divided in the joints, thus:

$$
\frac{\frac{1}{2} \pi\left(C_{o}^{4} C_{i}^{4}\right)}{C_{o}}=\frac{\sqrt{(81342 / 52 \times 3)^{2}+(245182 / 56 \times 3)^{2}}}{\frac{276}{4}}=\frac{C_{o}^{4} \quad 22 / 5^{4}}{C_{o}}=7150
$$

It should be noted that the coefficients 3 in the above relation are due to the oscillatory loads due to the circulation and due to the circumference of the outer axis and the diameter of the outer diameter should be consider than this value.

\subsection{Device Evaluation}

In this research, the effect of two factors, angular velocity in three levels of 3500, 4500 and $5500 \mathrm{rpm}$ and the radius of the rotation in two levels of 125 and $175 \mathrm{~mm}$ was investigated in order to quality of treated sesame oil. In this experiment, the cylinder's time was selected for a constant test and equal to 5 minutes. The amount of suspended solids in sesame oil was measured by means of a pictometer and then the data were analyzed by factorial experiment in a randomized complete block design with expert design software and modeled using response surface method.

\section{Results and Discussion}

In order to determine the quality of treated oil, the percentage of residual materials in sesame oil was measured. Based on the results, the radius of the rotation and cylinder's angular momentum on the gross particles in the refined oil was significant at $1 \%$ level, but the effect of repetition on them was not affected. Also, the interaction between the angular velocity of the cylinder and the radius of the nonsignificant (Table1).

Table 1. Analysis of variance of the percentage of solids remaining in refined oil.

\begin{tabular}{llll}
\hline Parameter & Df & Mean Square \\
\hline Rotation velocity of cylinder & 2 & 16.725 \\
Radius of rotation & 1 & 22.22 \\
Rotation velocity of cylinder $\times$ Radius of rotation & 2 & 0.724 & $24.1^{* *}$ \\
Duplication & 2 & 0.725 \\
Error & 10 & $0.785^{\text {n.s }}$ \\
\hline
\end{tabular}

Notic: ** Significant of $1 \%$ level, ns Non signidicant

Regarding the regression diagram (Figure 3), the adjusted $\mathrm{R}^{2}$ and predicted $\mathrm{R}^{2}$ values were very close and $92 \%$ and $91 \%$, respectively. The regression formula was also obtained from the formula 4 for prediction.

Substance $=$ Rotation velocity of cylinder $\times 0.0358$ - Radius of rotation $\times 0.0943+1.11$ (Rotation velocity of cylinder $\times$ Radius of rotation) +6.45

According to Figures 4, it can be claimed that the speed of centrifuges and the radius of the rotating cylinder are correlated with the amount of solids (debris) of sesame oil. Using a cylinder with a radius of $175 \mathrm{~mm}$, it was observed that the highest amount of pulp in the sesame seed oil was at 3500 and 7.4 percent, and the lowest amount of pulp in the 5500 and 4.4 percent, respectively.

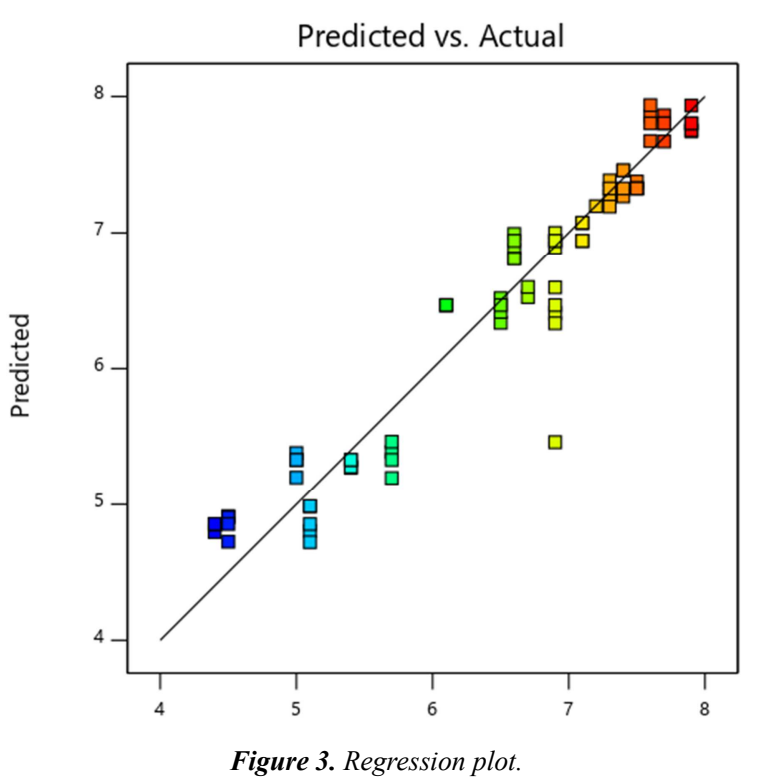


a
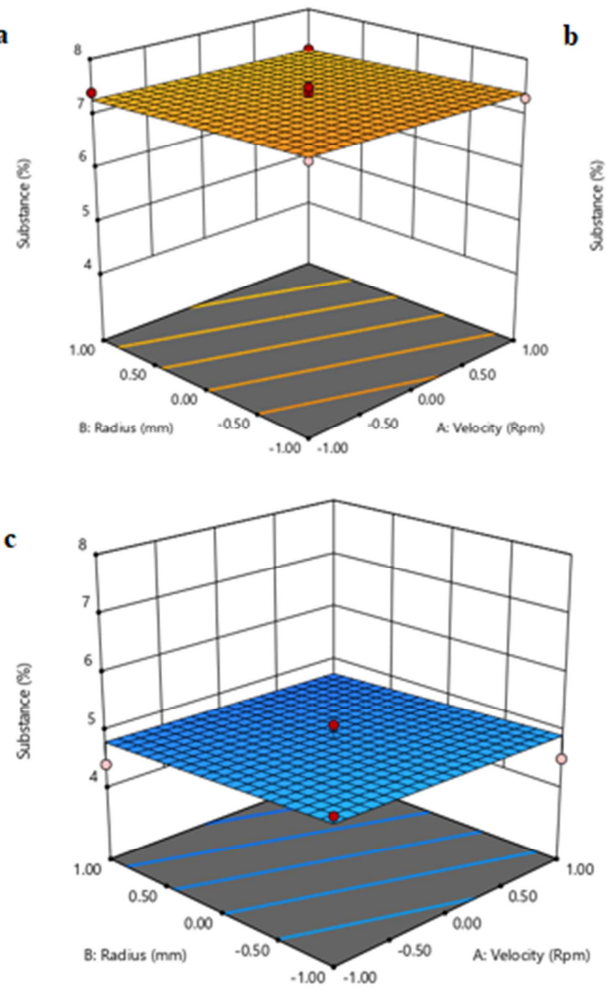

b
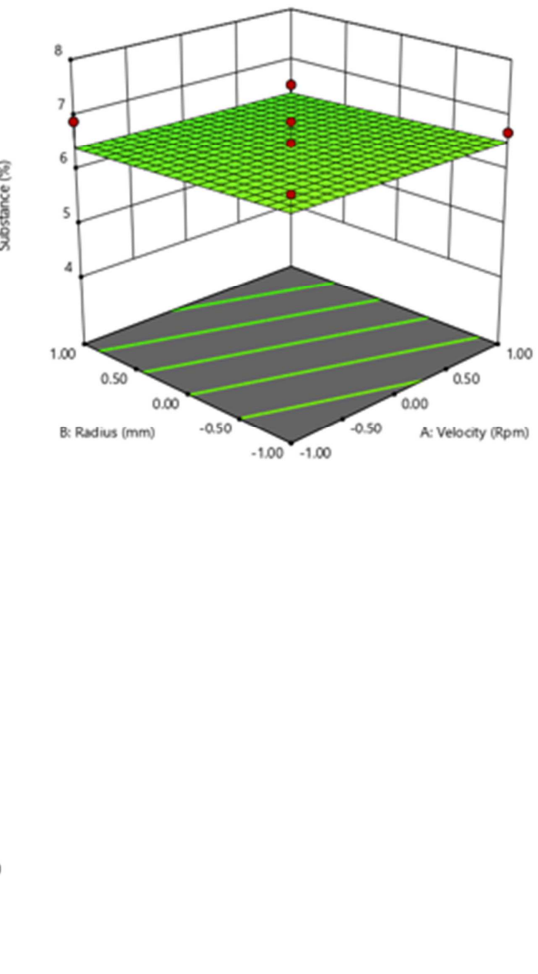

Figure 4. Response surface method for effect of various velocity of cylinder by radius level of $175 \mathrm{~mm}$ on substance value (a: $3500 \mathrm{rpm}$, b: $4500 \mathrm{rpm}$, c: 5500 rpm).

The reports were also correct for a $125-\mathrm{mm}$ cylinder. According to the figure, it can be concluded that the lowest and highest amount of pulp in the outlet oil was obtained in the cylinder at 5500 and 3500 , respectively, and equal to 5 and $7.9 \%$, respectively. With regard to the amount of solids output in sesame oil, the output of the machine can be concluded that the cylinder speed has a greater impact on the cylinder radius than the output oil quality.

$\mathbf{a}$
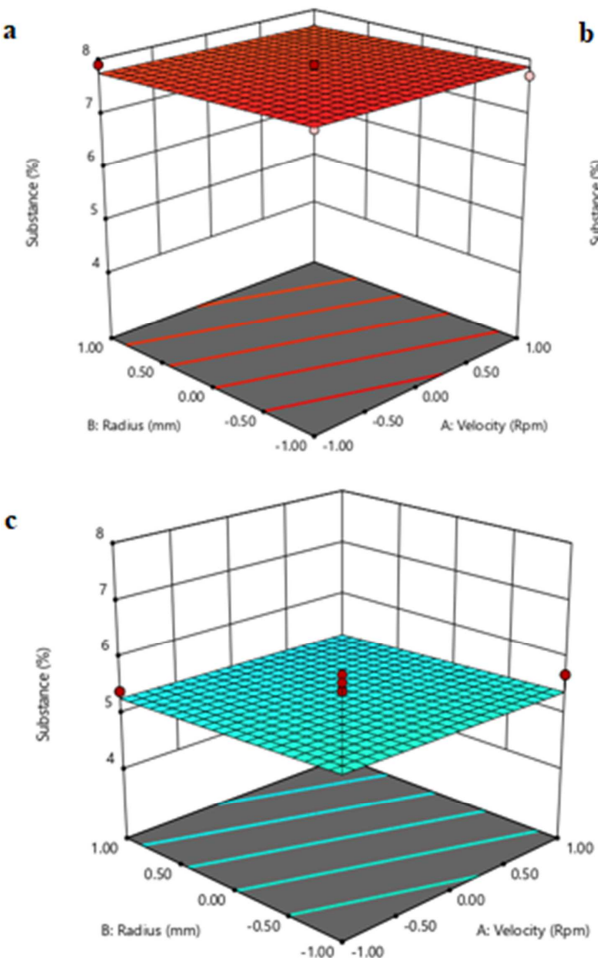

b

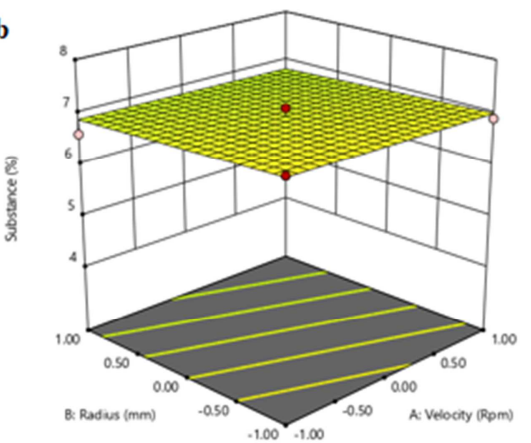

Figure 5. Response surface method for effect of various velocity of cylinder by radius level of $125 \mathrm{~mm}$ on substance value (a: $3500 \mathrm{rpm}, \mathrm{b}: 4500 \mathrm{rpm}, \mathrm{c}: 5500$ rpm). 
The method used in this study was similar to the methods for extracting sesame oil by Williams. In 2009, Williams et al. Claimed that in the continuous press machine, the pressure increase resulted in three ways of: reducing the output of the machine, reducing the size of the oil outlet and reducing the spiral rotational speed. In all cases, the increase in pressure increases the amount of oil extracted, while on the other hand, it reduces the feeding rate of the device and increases the power consumption, which should be chosen by studying the production costs and the amount of income to be the best [18]. Also, In a research that conducted by Jeon et al. about extraction sesame oil by chromatography, A sample containing $3 \mathrm{~g}$ of crude extract was consecutively injected four times onto the SCPC-1000, which yielded $328 \mathrm{mg}$ of sesamin and $168 \mathrm{mg}$ of sesamolin. These compounds were analyzed by highperformance liquid chromatography and determined to have purities of $95.6 \%$ and $93.9 \%$, respectively [19].

\section{Conclusion}

Increasing the velocity and radius of the cylinder in the rotary separator will result in better separation of the solids from the mixture of oil and lend from the seed presser machine. This is due to the rule of the Fugitive from the center of the fluid. In the centrifuge, the injection and removal of solids from the oil, as well as the evacuation of solids from the cylinder, and the preparation of the device for re-use are much less time-consuming than the filter press. It should be noted that the device made in this research is an appropriate replacement for some of the limitations, such as the need for a high cylinder to provide better separation of materials from one another, the size of the separation chamber (cylinder capacity) and the high speed dangers Chance of failure and throwing parts.

\section{Acknowledgements}

The authors would like to Machine design and Mechatronics Department, Institute of Mechanics, Iranian Research Organization for Science and Technology for their contributions to this study.

\section{References}

[1] Namiki, M. 2007. Nutraceutical functions of sesame: a review. Critical reviews in food science and nutrition, 47(7): 651-673. 2007.

[2] Schwertner, H. A., \& Rios, D. C. (2012). Analysis of sesamin, asarinin, and sesamolin by HPLC with photodiode and fluorescent detection and by GC/MS: application to sesame oil and serum samples. Journal of the American Oil Chemists' Society, 89(11), 1943-1950.

[3] Rohman, A., \& Man, Y. B. C. (2011). Palm oil analysis in adulterated sesame oil using chromatography and FTIR spectroscopy. European Journal of Lipid Science and Technology, 113(4), 522-527.
[4] Monteiro, É. M. H., Chibli, L. A., Yamamoto, C. H., Pereira, M. C. S., Vilela, F. M. P., Rodarte, M. P.,... \& da Luz André de Araújo, A. (2014). Antinociceptive and anti-inflammatory activities of the sesame oil and sesamin. Nutrients, 6(5), 19311944.

[5] Wu, R., Ma, F., Zhang, L., Li, P., Li, G., Zhang, Q. \& Wang, $X$. (2016). Simultaneous determination of phenolic compounds in sesame oil using LC-MS/MS combined with magnetic carboxylated multi-walled carbon nanotubes. Food chemistry, 204, 334-342.

[6] Zhuang, X., Han, M., Kang, Z. L., Wang, K., Bai, Y., Xu, X. L., \& Zhou, G. H. (2016). Effects of the sugarcane dietary fiber and pre-emulsified sesame oil on low-fat meat batter physicochemical property, texture, and microstructure. Meat science, 113, 107-115.

[7] RAJENDRA, K. PRODUCTION OF BIO-DIESEL FROM SESAME OIL USING ASPEN SIMULATION.

[8] Hashempour-Baltork, F., Torbati, M., Azadmard-Damirchi, S., \& Savage, G. P. (2017). Quality properties of sesame and olive oils incorporated with flaxseed oil. Advanced pharmaceutical bulletin, 7(1), 97.

[9] Carrín, M. E. and Crapiste, G. H. 2008. Mathematical modeling of vegetable oil-solvent extraction in a multistage horizontal extractor. J. Food Engineering. 85(3), 418-425. 2008.

[10] Mandal, V., Mohan, Y. and Hemalatha, S. 2007. Microwaveassisted extraction- an innovative and promising extraction tool for medicinal plant research. J. Pharmacognosy Reviews. 1(1), 7-18. 2007.

[11] Rodrigues, S. and Pinto, G. A. S. 2007. Ultrasound extraction of phenolic compounds from coconut (Cocos nucifera) shell powder. Journal of Food Engineering. 80, 869-872. 2007.

[12] Hemwimon, S., Pavasant, P. and Shotipruk, A. 2007. Microwave-assisted extraction of antioxidative anthraquinones from roots of Morinda citrifolia. Separation and Purification Technology. 54, 44-50. 2007.

[13] Japon-Lujan, R. and Luque de Castro, M. D. 2006. Superheated liquid extraction of oleuropein and related biophenols from olive leaves. Journal of Chromatography A. 1136: 185-191. 2006

[14] Pradhan, R. C., Mishra, S., Naik, S. N., Bhatnagar, N., Vijay, V. K. (2011). Oil expression from Jatropha seeds using a screw press expeller. J. Biosystems Engineering, 109, 2, 158166. 2001.

[15] Beerens, P. 2007. Screw-pressing of Jatropha seeds for fuelling purposes in less developed countries. M. Sc. Thesis, Department of Sustainable Energy Technology, Eindhoven University of Technology, Eindhoven, 87 p. 2007.

[16] Cerutti, M. L. M. N., Souza, A. A., Souza, S. M. 2012. Solvent extraction of vegetable oils: Numerical and experimental study. J. Food and Bioproducts Processing, 90, 2, 199-204. 2012.

[17] Sarkis, J. R., Boussetta, N., Tessaro, I. C., Marczak, L. D. F., \& Vorobiev, E. (2015). Application of pulsed electric fields and high voltage electrical discharges for oil extraction from sesame seeds. Journal of Food Engineering, 153, 20-27. 
[18] Willems, P., Kuipers, N. J. M., De Haan, A. B. 2009. A consolidation based extruder model to explore GAME process configurations. J. Food Eng, 90, 238-245. 2009.

[19] Jeon, J. S., Park, C. L., Syed, A. S., Kim, Y. M., Cho, I. J., \&
Kim, C. Y. (2016). Preparative separation of sesamin and sesamolin from defatted sesame meal via centrifugal partition chromatography with consecutive sample injection. Journal of Chromatography B, 1011, 108-113. 